\title{
Analytical approximation for the quantum-well gain and refractive-index spectra of vertical-cavity surface-emitting lasers including the effect of uniaxial planar stress
}

\author{
Guy Van der Sande, ${ }^{*}$ Jan Danckaert, Irina Veretennicoff, and Krassimir Panajotov ${ }^{\dagger}$ \\ Vrije Universiteit Brussel, Department of Applied Physics and Photonics (TW-TONA), Pleinlaan 2, 1050 Brussels, Belgium \\ Salvador Balle \\ Departamento de Física Interdisciplinar, Instituto Mediterráneo de Estudios Avanzados (Consejo Superior de Investigaciones Científicas \\ Universitat de les Illes Balears), E-07071 Palma de Mallorca, Spain
}

(Received 15 October 2004; published 2 June 2005)

\begin{abstract}
Starting from a microscopic model in the free-carrier approximation, we derive an analytical approximation for the optical susceptibility of uniaxially stressed quantum-well lasers at low temperatures by neglecting second-order contributions of the band-mixing phenomenon. The resulting polarization-dependent peak gains, differential peak gains, transparency carrier densities, and linewidth enhancement factors as induced by the uniaxial planar stress are discussed.
\end{abstract}

DOI: 10.1103/PhysRevA.71.063801

PACS number(s): 42.55.Px

\section{INTRODUCTION}

Vertical-cavity surface-emitting lasers (VCSELs) are touted as key components for present and future data communication systems. Due to their small cavity length and quasicylindrical symmetry, VCSELs have only one longitudinal mode, a superior beam quality, low power consumption, a long lifetime, and can be manufactured in twodimensional arrays. All these characteristics are often cited as substantial advantages over edge-emitting semiconductor lasers. However, their polarization behavior is difficult to predict due to their symmetrical structure. In the fundamental transverse-mode regime, VCSELs exhibit two nearly degenerate linear polarization modes with polarization direction along the [110] or [1 $\overline{1} 0]$ of the III-V based materials, between which switching has been observed when the current is changed. Different physical mechanisms have been introduced to explain this current-driven polarization switching (PS). The first model is of thermal nature and attributes PS to a spectral shift of the gain maximum with respect to the cavity resonances for the two frequency-split polarization modes [1]. Some of us have further elaborated on this idea and have incorporated the temperature and frequency dependence of both losses and gain [2]. Furthermore, the gain differences that induce PS have been attributed to thermal lensing in [3] or to the overlap of the modal profiles with the carrier distribution in the device [4]. Nonlinear gain contributions, so-called gain saturation effects, can also be involved in PS $[5,6]$. These nonlinearities can stem from spatial hole burning effects [7], spectral hole burning effects [8-10], intersubband absorption [11], and carrier heating $[12,13]$.

A distinctively different model for PS was developed by San Miguel et al. [14] and was extended to include fre-

\footnotetext{
*Electronic address: guy.van.der.sande@vub.ac.be

${ }^{\dagger}$ Also at Institute of Solid State Physics, 72 Tzarigradsko Chaussee Blvd., 1784 Sofia, Bulgaria.
}

quency and gain anisotropies in [15]. The latter model, called the spin-flip model (SFM), describes the active semiconductor quantum well in terms of a spin-split two level system, where the two spin subsystems are coupled through spin-flip processes.

In $[16,17]$, the SFM was extended by introducing an approximate frequency-dependent susceptibility for quantumwell $(\mathrm{QW})$ media. This approach permits one to include the thermal shift of the cavity resonances over the gain spectrum of the system, and has allowed reproduction of the experimental observations of PS [17,18], two-frequency emission at threshold [19], and the emission of elliptically polarized states [20]. In all these modeling attempts, strain effects are introduced phenomenologically through the dichroism and birefringence parameters. These parameters, which describe the residual anisotropies in the structure that may result from unintentional residual strain left after the growth process or from the electro-optic or elasto-optic effects in the VCSEL cavity [21] play a key role in the preference for a particular polarization orientation.

Indeed, in [22,23] it was shown that mechanical stress, externally applied to the VCSEL package, dramatically alters its polarization behavior. We considered a VCSEL operating at the high-frequency side of the gain spectrum, so that at threshold, the laser selects the low-frequency mode that keeps lasing at every current above threshold. We then applied tensile stress and considered two cases: stress was ap-

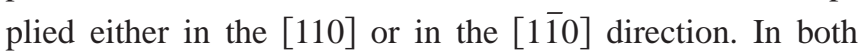
cases, the laser switches to the high-frequency mode (polarized perpendicular to the stress axis) at high switching current. This switching current drops upon increasing the stress. At lasing threshold, only the high-frequency mode lases. The present paper aims at understanding this impact of in-plane anisotropic stress on the quantum-well gain spectra.

Our starting point to study the effect of in-plane anisotropic stress on the gain spectrum of GaAs quantum wells was the Luttinger-Kohn Hamiltonian for the quantum-well heavy-hole and light-hole bands and the introduction of the strain contributions according to the Bir-Pikus theory 
TABLE I. The material parameters.

\begin{tabular}{cccc}
\hline \hline & & & \\
& & $\mathrm{In}_{0.2} \mathrm{Ga}_{0.8} \mathrm{As}$ & $\mathrm{GaAs}$ \\
\hline$E_{g}(\mathrm{eV})$ & Band gap energy & 1.13 & 1.42 \\
$1 / \gamma$ & Conduction band effective mass & 0.057 & 0.067 \\
$\gamma_{1}$ & Luttinger parameter & 8.09 & 6.98 \\
$\gamma_{2}$ & Luttinger parameter & 2.69 & 2.06 \\
$\mathrm{VBO}(\mathrm{eV})$ & Valence band offset & -0.70 & -0.80 \\
$E_{0 e}(\mathrm{meV})^{\mathrm{a}}$ & Electron confinement energy & 44.1 & $\ldots$ \\
$E_{0 h h}(\mathrm{meV})^{\mathrm{b}}$ & Electron confinement energy & 10.2 & $\ldots$ \\
$E_{0 l h}(\mathrm{meV})^{\mathrm{c}}$ & Electron confinement energy & 29.6 & $\ldots$ \\
\hline \hline
\end{tabular}

${ }^{\mathrm{a} e} \equiv$ electron

${ }^{\mathrm{b}} h h \equiv$ heavy hole.

${ }^{\mathrm{c}} \mathrm{lh} \equiv$ light hole.

$[24,25]$. The numerical studies by Burak et al. $[26,27]$ have provided valuable insight into the experimental results obtained in [22], demonstrating the changes induced in the gain spectrum of the QW by the application of stress. However, these numerical studies are complex and demanding from the computational point of view. This fact stimulated the search for simpler, analytical studies of the optical gain.

Here, we present an analytical study of the optical susceptibility of a quantum-well laser at low temperature taking stress effects into account. This work is performed in line with the spirit of [16]. Its primary objective is to develop a susceptibility function which retains the key features of the semiconductor media including the changes due to stress. Our analysis is based on a parabolic-band approach, with the assumption that second-order contributions of the bandmixing effects can be neglected. All parameters can be determined from the band structure, except for the band gap renormalization.

The paper is organized as follows. In Sec. II, we present the model for the electronic band structure and the optical susceptibility tensor of a stressed semiconductor quantum well. In Sec. III, we gain more insight into the effect of the stress-induced symmetry change in the system. This leads to a stress-induced band mixing contribution, which is crucial to explain the alignment of the eigenmodes with the stress directions. The first approximation is made by neglecting second-order contributions of the band mixing. In Sec. IV, the low-temperature susceptibility is reduced to an analytical formula using a parabolic band approach. In Secs. V and VI, this analytical expression is discussed by studying the effect of uniaxial planar stress on the spectra of the quantum-well gain and refractive index. Section VII contains conclusions.

\section{BAND STRUCTURE AND OPTICAL SUSCEPTIBILITY OF STRESSED QW SEMICONDUCTORS}

We consider a $8 \mathrm{~nm} \operatorname{In}_{0.2} \mathrm{Ga}_{0.8}$ As quantum well sandwiched between GaAs (see Table I: all band parameters are taken from [28]). We have chosen the parameters such that the one-bound-state approximation can be made. By this we mean that we disregard all the states confined in the quantum well except those with the lowest energy within each band.
In order to study the effects of band mixing and stress, it is convenient to write the Hamiltonian on the basis of the energy eigenstates $|\mathbf{k} h \pm\rangle$ and $|\mathbf{k} l \pm\rangle$, rather than using the angular momentum states $| \pm 3 / 2\rangle$ and $| \pm 1 / 2\rangle$. The heavy- and light-hole states, $|\mathbf{k} h \pm\rangle$ and $|\mathbf{k} l \pm\rangle$, coincide with the pure angular momentum states, $| \pm 3 / 2\rangle$ and $| \pm 1 / 2\rangle$, only at the band edge. Due to band mixing, the energy eigenstates will be linear combinations of the angular momentum eigenstates when the carrier momentum $\mathbf{k}$ differs from zero. This change of basis is performed in Appendix A. In this case, the Hamiltonian of the QW system, including the interaction with a semiclassical external optical field $\mathbf{E}$, reads

$$
\begin{aligned}
\hat{H}= & \sum_{\mathbf{k}, s} E^{c}(\mathbf{k}) a_{\mathbf{k}, s}^{\dagger} a_{\mathbf{k}, s}+\sum_{\mathbf{k}, \sigma} E^{\sigma}(\mathbf{k})\left(c_{\mathbf{k}, \sigma+}^{\dagger} c_{\mathbf{k}, \sigma+}+c_{\mathbf{k}, \sigma-}^{\dagger} c_{\mathbf{k}, \sigma-}\right) \\
& -\hat{\mathbf{P}} \cdot \mathbf{E}, \\
\hat{\mathbf{P}}= & \sum_{\mathbf{k}}\left(\mathbf{d}_{\mathbf{k}, h+} a_{\mathbf{k}, 1 / 2}^{\dagger} c_{\mathbf{k}, h+}^{\dagger}+\mathbf{d}_{\mathbf{k}, l-l} a_{\mathbf{k}, 1 / 2}^{\dagger} c_{\mathbf{k}, l-}^{\dagger}+\mathbf{d}_{\mathbf{k}, l+l} a_{\mathbf{k},-1 / 2}^{\dagger} c_{\mathbf{k}, l+}^{\dagger}\right. \\
& \left.+\mathbf{d}_{\mathbf{k}, h-} a_{\mathbf{k},-1 / 2}^{\dagger} c_{\mathbf{k}, h-}^{\dagger}\right)+ \text { h.a. }
\end{aligned}
$$

where h.a. denotes the hermitian adjoint. $a_{\mathbf{k}, s}\left(a_{\mathbf{k}, s}^{\dagger}\right)$ is the annihilation (creation) operator for electrons in the conduction band states $|\mathbf{k} s\rangle$ with in-plane wave vector $\mathbf{k}$, and spin $s$ $= \pm 1 / 2$, which have energies $E^{c}(k)$ given by

$$
E^{c}(k)=E_{g}+E_{0 e}^{\prime}+\left(\hbar^{2} \gamma / 2 m_{0}\right) k^{2}+A_{e}\left(\sigma_{[110]}+\sigma_{[110]}\right),
$$

where $E_{g}$ is the gap energy, $E_{0 e}^{\prime}$ is the conduction band offset, and $m_{0} / \gamma$ is the effective mass in the conduction band states. Analogously, $c_{\mathbf{k}, \sigma \pm}\left(c_{\mathbf{k}, \sigma \pm}^{\dagger}\right)$ is the corresponding annihilation (creation) operator for holes in the valence band with $\sigma$ $=h, l$ for the heavy- and the light-hole band. The heavy-hole and light-hole energies, $E^{h}(\mathbf{k})$ and $E^{l}(\mathbf{k})$, respectively, are the eigenenergies of the Luttinger Hamiltonian, which (in the basis of the electronic angular momentum eigenstates at band edge) reads [24]

$$
T=\left(\begin{array}{cccc}
H^{h} & C & 0 & 0 \\
C^{*} & H^{l} & 0 & 0 \\
0 & 0 & H^{l} & C \\
0 & 0 & C^{*} & H^{h}
\end{array}\right) .
$$

It is worth noting that in the experiments, tensile stress is applied to the VCSEL structure in the [110] and [1 $\overline{10}]$ directions $\left(\sigma_{[110]}\right.$ and $\sigma_{[1 \overline{1} 0]}$, respectively). In addition, there is some residual stress $\sigma_{z}$ present in the growth direction ([001]). Therefore, the study of the effects of this externally (or possibly internally) applied stress, requires the rewriting of the Hamiltonian in such a way that the dependencies on the stress tensor are made clear (see Appendix A). The elements of the Luttinger Hamiltonian are

$$
\begin{aligned}
& H^{h}(k)=E_{0 h h}^{\prime}+\frac{\hbar^{2}}{2 m_{0}}\left(\gamma_{1}+\gamma_{2}\right) k^{2}+A_{h}\left(\sigma_{[110]}+\sigma_{[1 \overline{10}]}\right), \\
& H^{l}(k)=E_{0 l h}^{\prime}+\frac{\hbar^{2}}{2 m_{0}}\left(\gamma_{1}-\gamma_{2}\right) k^{2}+A_{l}\left(\sigma_{[110]}+\sigma_{[1 \overline{10}]}\right),
\end{aligned}
$$


TABLE II. The stress-related material parameters.

\begin{tabular}{ccc}
\hline \hline$a_{e}(\mathrm{eV})$ & Hydrostatic deformation potential & -6.33 \\
$a_{1}(\mathrm{eV})$ & Hydrostatic deformation potential & -1.13 \\
$a_{2}(\mathrm{eV})$ & Shear deformation potential & -0.98 \\
$a_{3}(\mathrm{eV})$ & Shear deformation potential & -4.56 \\
$S_{11}\left(10^{-11} \mathrm{~m}^{2} / \mathrm{N}\right)$ & Compliance tensor element & 1.26 \\
$S_{12}\left(10^{-11} \mathrm{~m}^{2} / \mathrm{N}\right)$ & Compliance tensor element & -0.46 \\
$S_{44}\left(10^{-11} \mathrm{~m}^{2} / \mathrm{N}\right)$ & Compliance tensor element & 1.79 \\
$A_{e}\left(10^{-11} \mathrm{eV} \mathrm{m}^{2} / \mathrm{N}\right)$ & Stress potential & -2.84 \\
$A_{h}\left(10^{-11} \mathrm{eV} \mathrm{m} / \mathrm{N}\right)$ & Stress potential & -2.14 \\
$A_{l}\left(10^{-11} \mathrm{eV} \mathrm{m}^{2} / \mathrm{N}\right)$ & Stress potential & 1.13 \\
$A_{c}\left(10^{-11} \mathrm{eV} \mathrm{m}^{2} / \mathrm{N}\right)$ & Stress potential & -7.06 \\
\hline \hline
\end{tabular}

$$
C(k)=-\frac{\sqrt{3} \hbar^{2}}{2 m_{0}}\left[\gamma_{2}\left(k_{x}^{2}-k_{y}^{2}\right)-2 i \gamma_{3} k_{x} k_{y}\right]+i A_{c}\left(\sigma_{[110]}-\sigma_{[1 \overline{10}]}\right)
$$

$x, y$, and $z$ denote the main crystallographic axes whose orientations define the [100], [010], and [001] directions of the III-V semiconductor crystal, respectively. The effect of $\sigma_{z}$ is included in $E_{0 e}^{\prime}, E_{0 h h}^{\prime}$, and $E_{0 l h}^{\prime}$ (see Appendix A), making clear that its role is simply to shift the edges of the bands. The stress potentials $A_{e, h, l, c}$ are defined in Appendix A. The stress- and strain-related material parameters are summarized in Table II (parameters taken from [28]).

The valence and conduction band states are coupled when an optical field is applied; assuming momentum conservation, the interaction among them is described by $\mathbf{d}_{\mathbf{k}, \sigma \pm}$, the matrix element of the electric dipole moment. Since the cavity axis is parallel to the quantization axis and the basis states are eigenstates of the $z$-component of the angular momentum, the optical transitions between the valence and conduction bands involve combinations of left- and right-circularly polarized light, whose polarization state is $\mathbf{e}_{ \pm}$ $=\mp\left(\mathbf{e}_{x} \pm i \mathbf{e}_{y}\right) / \sqrt{2}$. These selection rules impose that the only nonvanishing dipole moments are

$$
\begin{aligned}
& \mathbf{d}_{\mathbf{k}, h+}=\gamma_{\mathbf{k}} \mu\left(\mathbf{e}_{+}+\frac{\beta_{\mathbf{k}}^{*}}{\sqrt{3}} \mathbf{e}_{-}\right), \\
& \mathbf{d}_{\mathbf{k}, l-}=\gamma_{\mathbf{k}} \mu\left(-\beta_{\mathbf{k}} \mathbf{e}_{+}+\frac{\mathbf{e}_{-}}{\sqrt{3}}\right) \text {, } \\
& \mathbf{d}_{\mathbf{k}, l+}=\gamma_{\mathbf{k}} \mu\left(\frac{\mathbf{e}_{+}}{\sqrt{3}}-\beta_{\mathbf{k}}^{*} \mathbf{e}_{-}\right), \\
& \mathbf{d}_{\mathbf{k}, h-}=\gamma_{\mathbf{k}} \mu\left(\frac{\beta_{\mathbf{k}}}{\sqrt{3}} \mathbf{e}_{+}+\mathbf{e}_{-}\right),
\end{aligned}
$$

with

$$
\beta_{\mathbf{k}}=\frac{C^{*}}{E^{h}-H^{l}}=\frac{C^{*}}{H^{h}-E^{l}}
$$

$$
\gamma_{\mathrm{k}}=\frac{1}{\sqrt{1+\left|\beta_{\mathrm{k}}\right|^{2}}} .
$$

where $\beta_{\mathbf{k}}$ is a measure for the strength of the band mixing.

Due to the presence of the optical field $\mathbf{E}$, the otherwise eigenstates of Eq. (1) evolve in time according to Heisenberg's equation, thus determining the optical response of the system in the one-bound-state approximation. In the general case, the optical response of the QW medium includes the simultaneous contribution of the heavy-hole and light-hole states. The calculation of the optical susceptibility becomes quite involved due to band mixing and the associated coherence among the states in the valence band. These difficulties can be overcome by considering those strained QW systems where, due to the large energy splitting between the heavyhole and the light-hole band as compared to the linewidth of a quasimonochromatic field, the light-hole band does not contribute to the optical susceptibility. This approximation, which is valid for GaAs QW media operating at $980 \mathrm{~nm}$ and beyond, allows for a qualitative picture of the effects of strain on the optical response of the system. It permits a first evaluation and understanding of the effects of strain and stress on the gain and refractive index spectra of the active medium.

Since the mixed heavy-hole and light-hole states have well separated energy levels and because we consider the interaction with a quasimonochromatic field, we can consider the case where the optical field only couples the heavyhole states $|h \pm\rangle$ to the conduction band. In Appendix B, the optical susceptibility is obtained in the slowly varying envelope and rotating wave approximation when many-body effects are neglected. By using the standard definition of the optical susceptibility $\mathbf{P}=\varepsilon_{0} \overline{\bar{\chi}} \mathbf{E}$, we can identify the optical susceptibility $\overline{\bar{\chi}}$ from Eqs. (B12)-(B15) [written in the circular base $\left.\left(\mathbf{e}_{+}, \mathbf{e}_{-}\right)\right]$:

$$
\overline{\bar{\chi}}(\omega)=\left(\begin{array}{cc}
G_{-}(\omega)+H_{+}(\omega) & S_{+}(\omega)+S_{-}(\omega) \\
\hat{S}_{+}(\omega)+\hat{S}_{-}(\omega) & G_{+}(\omega)+H_{-}(\omega)
\end{array}\right),
$$

where we have defined

$$
\begin{gathered}
G_{ \pm}(\omega)=-\frac{i \mu^{2}}{\varepsilon_{0} \hbar V} \sum_{\mathbf{k}}\left|\gamma_{\mathbf{k}}\right|^{2} \frac{f_{\mathbf{k}, h \pm}+f_{\mathbf{k}, \pm 1 / 2}^{e}-1}{\gamma_{\perp}+i\left(\omega_{\mathbf{k}}^{h}-\omega\right)}, \\
H_{ \pm}(\omega)=-\frac{i \mu^{2}}{\varepsilon_{0} \hbar V} \sum_{\mathbf{k}}\left|\gamma_{\mathbf{k}}\right|^{2} \frac{f_{\mathbf{k}, h \pm}+f_{\mathbf{k}, \pm 1 / 2}^{e}-1}{\gamma_{\perp}+i\left(\omega_{\mathbf{k}}^{h}-\omega\right)} \frac{\left|\beta_{\mathbf{k}}\right|^{2}}{3}, \\
S_{ \pm}(\omega)=-\frac{i \mu^{2}}{\varepsilon_{0} \hbar V} \sum_{\mathbf{k}}\left|\gamma_{\mathbf{k}}\right|^{2} \frac{f_{\mathbf{k}, h \pm}+f_{\mathbf{k}, \pm 1 / 2}^{e}-1}{\gamma_{\perp}+i\left(\omega_{\mathbf{k}}^{h}-\omega\right)} \frac{\beta_{\mathbf{k}}^{*}}{\sqrt{3}}, \\
\hat{S}_{ \pm}(\omega)=-\frac{i \mu^{2}}{\varepsilon_{0} \hbar V} \sum_{\mathbf{k}}\left|\gamma_{\mathbf{k}}\right|^{2} \frac{f_{\mathbf{k}, h \pm}+f_{\mathbf{k}, \pm 1 / 2}^{e}-1}{\gamma_{\perp}+i\left(\omega_{\mathbf{k}}^{h}-\omega\right)} \frac{\beta_{\mathbf{k}}}{\sqrt{3}},
\end{gathered}
$$

with $f_{\mathbf{k}, \pm 1 / 2}^{e}$ and $f_{\mathbf{k}, h \pm}$ the Fermi distributions for electrons and holes, respectively, and $\gamma_{\perp}$ the dephasing rate of the dipoles due to intraband carrier-carrier scattering. The transition frequencies are defined by $\hbar \omega_{\mathbf{k}}^{h}=E^{c}(k)+E^{h}(\mathbf{k})$. 
From Eq. (14), it can be seen that there exist different contributions to the total optical susceptibility. The first contributions are the unmixed susceptibility functions $G_{ \pm}$. They describe the interaction between the circularly polarized fields and their respective spin channels in the absence of any band mixing; i.e., for $\beta_{\mathbf{k}}=0$. The other contributions describe the effects of band mixing on the optical susceptibility of the $\mathrm{QW}$, and they are of two different types. The first one, given by $H_{ \pm}$, describes how the changes in the electronic structure due to band mixing modify the optical response of the electronic states. The second, $S_{ \pm}$and $\hat{S}_{ \pm}$, make the susceptibility nondiagonal, expressing that due to band mixing, the leftand right-circularly polarized components are coupled to each other; therefore, these terms describe the stress-induced birefringence and dichroism in the optical response of the QW medium. In the absence of stress, Eqs. (17) and (18) are zero; as a result, the preferred base of the system is the circularly polarized one, the response for the two circular components being the same. However, when stress is applied to the device, the coupling between both circularly polarized fields is no longer negligible. This results in a new base of eigenmodes for the system dependent on the direction of the applied stress. For the experimental situation studied in [22], it resulted in a preference for the linear polarizations along [110] and $[1 \overline{1} 0]$.

\section{BAND MIXING IN A STRESSED QW}

Equations (14)-(18) determine the optical response of the QW medium. The effects of strain-induced band mixing on the optical susceptibility appear in Eqs. (15)-(18) through the function $\beta_{\mathbf{k}}$. This function can be split in a natural band mixing part $\beta_{\mathbf{k}, N}$, and in a strain-induced part $\beta_{\sigma}$, where

$$
\begin{gathered}
\beta_{\mathbf{k}, N}=\frac{-\left(\sqrt{3} \hbar^{2} / 2 m_{0}\right)\left[\gamma_{2}\left(k_{x}^{2}-k_{y}^{2}\right)+2 i \gamma_{3} k_{x} k_{y}\right]}{E^{h}-H^{l}}, \\
\beta_{\sigma}=-i \frac{A_{c}\left(\sigma_{[110]}-\sigma_{[110]}\right)}{E^{h}-H^{l}} .
\end{gathered}
$$

It is worth noting that, due to the large splitting between the heavy-hole and light-hole bands, the influence of the mixing terms is strongly reduced; i.e., the effects of band mixing can be included perturbatively. Up to first order in $\beta_{\mathrm{k}}$, the susceptibility tensor (in the circular base) has the form

$$
\overline{\bar{\chi}}(\omega)=\left(\begin{array}{cc}
G_{-}(\omega) & S_{+}(\omega)+S_{-}(\omega) \\
-S_{+}(\omega)-S_{-}(\omega) & G_{+}(\omega)
\end{array}\right),
$$

where $G_{ \pm}$is given in Eq. (15), $H_{ \pm}$has been neglected because it is a second-order quantity in $\beta_{\mathrm{k}}$, and

$$
S_{ \pm}(\omega)=-\frac{i \mu^{2}}{\varepsilon_{0} \hbar V} \sum_{\mathbf{k}} \frac{f_{\mathbf{k}, h \pm}+f_{\mathbf{k}, \pm 1 / 2}^{e}-1}{\gamma_{\perp}+i\left(\omega_{\mathbf{k}}^{h}-\omega\right)} \frac{\beta_{\sigma}^{*}}{\sqrt{3}}=-\hat{S}_{ \pm}(\omega) .
$$

Moreover, the heavy-hole energy is simply given by $E^{h}$ $\approx H^{h}=E_{0 h h}^{\prime}+\left(\hbar^{2} / 2 m_{0}\right)\left(\gamma_{1}+\gamma_{2}\right) k^{2}+A_{h}\left(\sigma_{[110]}+\sigma_{[1 \overline{10}]}\right)$; hence,

$$
\hbar \omega_{\mathbf{k}}^{h}=E^{c}(k)+E^{h}(k) \approx E_{t}+\frac{\hbar^{2} k^{2}}{2 m_{0}}\left(\gamma+\gamma_{1}+\gamma_{2}\right) \equiv E_{t}+\frac{\hbar^{2} k^{2}}{2 m},
$$

where the minimum transition energy is

$$
E_{t}=E_{g}+E_{0 e}+E_{0 h h}^{\prime}+\left(A_{e}+A_{h}\right)\left(\sigma_{[110]}+\sigma_{[1 \overline{0}]}\right) .
$$

Thus, since in our case the hydrostatic potentials $A_{e}$ and $A_{h}$ are negative (see Table II), the stress dependence of the minimum transition frequency will eventually lead to a redshift of the gain spectrum.

It is noteworthy that all the effects of natural band mixing have disappeared because $\beta_{\mathrm{k}, N}$ is antisymmetric upon exchange of $k_{x}$ and $k_{y}$ while the other terms depend only on $k^{2}$. Hence, the first order contributions involving $\beta_{\mathbf{k}, N}$ vanish upon summation over the Brillouin zone and the transition probabilities become constant, effectively simplifying the problem.

\section{ANALYTICAL APPROXIMATION TO STRESS- DEPENDENT OPTICAL SUSCEPTIBILITY}

Neglecting the second-order band-mixing effects in Eqs. (14)-(18) results in a great simplification of the optical susceptibility of the QW medium. Yet, the summations over the first Brillouin zone that define the different contributions cannot be performed analytically in the general case. However, we can consider the low-temperature limit as in [16], which has proven to give a useful qualitative description of the optical response. This approximation provides us with a qualitative picture of the effects of strain on the optical response of the system that allows us to understand more easily the effects of strain and stress on the gain and refractive index spectra of the active medium. This approximation allows to describe the optical response of the system in dependence of the densities of electrons in each spin orientation normalized to the transparency carrier density $D_{ \pm}$. We can approximate the susceptibility tensor as follows (see Appen$\operatorname{dix} \mathrm{C})$ :

$$
\overline{\bar{\chi}}\left(D_{+}, D_{-}, u\right)=\left(\begin{array}{cc}
G_{-}(u) & S_{+}(u)+S_{-}(u) \\
-S_{+}(u)-S_{-}(u) & G_{+}(u)
\end{array}\right),
$$

with

$$
\begin{gathered}
G_{ \pm}(u)=-\frac{\chi_{0}}{2} \ln \left(\frac{\left(u+i-2 D_{ \pm}\right)\left(u+i-D_{+}-D_{-}\right)}{(u+i)(u+i-b)}\right), \\
S_{ \pm}(u)=i S_{0} \frac{\sigma_{[110]}-\sigma_{[11 \overline{1} 0]}}{u-u_{\sigma}}\left[G_{ \pm}(u)-G_{ \pm}\left(u_{\sigma}\right)\right] .
\end{gathered}
$$

with

$$
u=\Delta+\rho\left(D_{+}+D_{-}\right)^{1 / 3},
$$

where $\Delta=\left(\omega-\omega_{t}\right) / \gamma_{\perp}$ is the detuning between the optical frequency $\omega$ and the nominal transition frequency $\omega_{t}$ normalized to the linewidth, and $\rho$ describes phenomenologically the effective band-gap shrinkage with the total carrier density. $b$ measures, in units of $\gamma_{\perp}$, the total energy spread where 
TABLE III. The model parameters.

\begin{tabular}{ccc}
$S_{0}\left(10^{-8} \mathrm{~m}^{2} / \mathrm{N}\right)$ & Gain splitting strength & -3.25 \\
$b$ & Reduced total energy spread & $2 \times 10^{4}$ \\
$u_{\sigma 0}$ & Reduced intraband energy gap & -15.42 \\
$u_{\sigma 1}\left(10^{-8} \mathrm{~m}^{2} / \mathrm{N}\right)$ & Reduced stress-induced & -2.60 \\
& intraband energy gap & \\
$\rho$ & Band-gap renormalization & 0.2 \\
\hline \hline
\end{tabular}

optical transitions from the the heavy hole and to the conduction band may occur, and

$$
\begin{aligned}
u_{\sigma} & =-i-\frac{\gamma+\gamma_{1}+\gamma_{2}}{2 \gamma_{2}} \frac{E_{0 h h}^{\prime}-E_{0 l h}^{\prime}+\left(A_{h}-A_{l}\right)\left(\sigma_{[110]}+\sigma_{[110]}\right)}{\hbar \gamma_{\perp}} \\
& =-i-u_{\sigma 0}-u_{\sigma 1}\left(\sigma_{[110]}+\sigma_{[1 \overline{10}]}\right)
\end{aligned}
$$

which corresponds to the reduced intraband energy gap. All model parameters are summarized in Table III and their definitions can be found in Appendix C.

In a steady state with equal spin populations $D_{ \pm}=D$, $G_{ \pm}(u)=G(u, D)$ and $S_{ \pm}(u)=S(u, D)$. Hence the susceptibility tensor can be diagonalized in the base $\left( \pm i E_{+}+E_{-}\right) / \sqrt{2}$,

$$
\overline{\bar{\chi}}(u, D)=\left(\begin{array}{cc}
G(u, D)+i 2 S(u, D) & 0 \\
0 & G(u, D)-i 2 S(u, D)
\end{array}\right) .
$$

This base corresponds to ([1] $\overline{10}],[110])$. Thus, the externally applied stress in the [1피 or [110], transforms the eigensystem of the optical interaction of the quantum well, to overlap with the direction in which the stress is applied. Indeed, this result does not rely on the first-order limit, but only on the equality of the occupancies for the two spin orientations and the antisymmetric character of $\beta_{\mathbf{k}}$ : when stress is applied along the direction of the crystallographic axes of [110], $[1 \overline{10}],[100]$, or [010], the eigenmodes will always be transformed to a linearly polarized base with appropriate directions. For a stress direction that is in between, the eigenmodes will in general be elliptically polarized. On the other hand, in the same limit, the resulting modal susceptibilities are formally the same. We would like to point out that, within the SFM, when the spin-flip rate is very high, the difference $D_{+}-D_{-}$relaxes to zero on very fast time scales, leading to equal spin populations even when the system is not in a steady state. In this case, the diagonalization to the linearly polarized base determined by the applied stress can be always performed.

\section{MATERIAL GAIN SPECTRUM}

In the following sections, we will always assume that a steady state is reached such that $D_{+}=D_{-}=D$. In that case, the susceptibility tensor can be diagonalized in a linearly polarized base along the crystallographic axes [110] and [1 $\overline{10}]$.
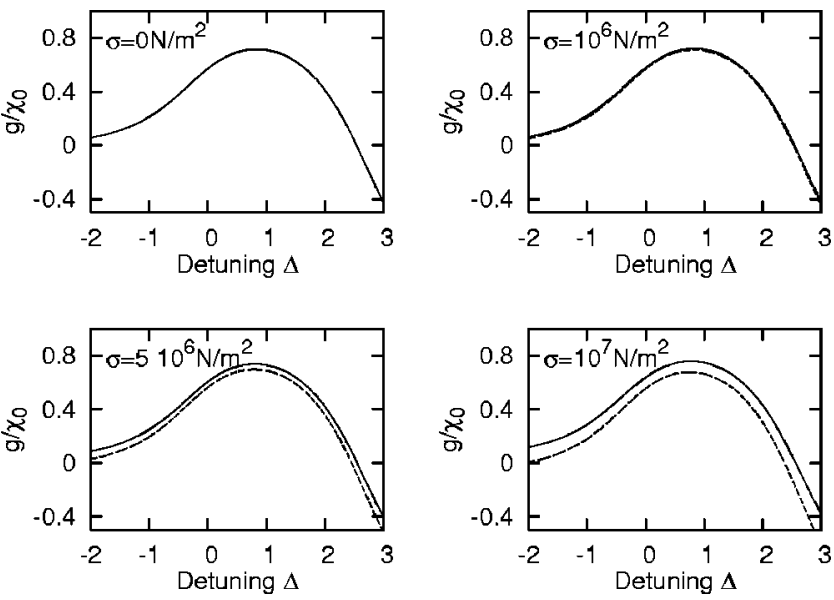

FIG. 1. Gain spectra as a function of detuning for different values of $\sigma_{[110]} \cdot g_{[1 \overline{10}]}$ is plotted with solid lines, while $g_{[110]}$ is dashed. $D=1.5$ and all other parameters are as in Table III.

Moreover, due to the carrier-induced band-gap shrinkage, we will express the frequency dependence of the optical response with $\Delta$ instead of $u$. The material gain spectrum is determined from the imaginary part of the susceptibility as

$$
\begin{aligned}
& g_{[110]}(D, \Delta)=-\operatorname{Im}[G(D, \Delta)]+2 \operatorname{Re}[S(D, \Delta)], \\
& g_{[1 \overline{10}]}(D, \Delta)=-\operatorname{Im}[G(D, \Delta)]-2 \operatorname{Re}[S(D, \Delta)] .
\end{aligned}
$$

In Fig. 1, we plot the gain spectra, both for linearly polarized light along the [110] (dashed) and [1̄ㅣㄹ (solid) directions, for different values of the tensile stress $\sigma_{[110]}$ in the [110] direction. The characteristics of the gain spectrum with a zero stress have been extensively studied and commented on in [16]. When stress is applied in the [110] direction, the two gain spectra split with a higher gain for the [1히 polarized electric field and a lower for the field polarized in [110]. This result is in agreement with the experiments of [22], where the mode favored by the tensile stress has a polarization direction orthogonal to the direction of stress. Qualitatively, this result can be explained by inspection of the dipole moments $d_{\mathbf{k}, h \pm}$ [in Eqs. (8) and (11)] under uniaxial stress. Due to the stress-induced band mixing, applying stress in the [110] direction reduces the dipole moment along [110], while enlarging it in the orthogonal direction. Thus, the mode polarized orthogonally to the stress direction can be expected to have the higher gain. This stress-induced change in the dipole matrix elements is modeled by the function $S$ in Eq. (31). In addition, a shift of the gain peak is noticeable. This shift of the gain peak and the gain splitting suggest to study the change in gain peak position, in peak gain, and in transparency carrier density.

We have plotted the position of the gain peak $\Delta_{p}$ in Fig. 2(a). Our observation of the shift of the gain maximum is confirmed. The gain related to the electric field polarized in the $[1 \overline{1} 0]$ direction (solid lines) has its peak at lower detuning with increasing stress. The same holds for the perpendicular direction but at a faster pace. We study the gain peak in Fig. 2(b). Again, our observation is confirmed. The gain 

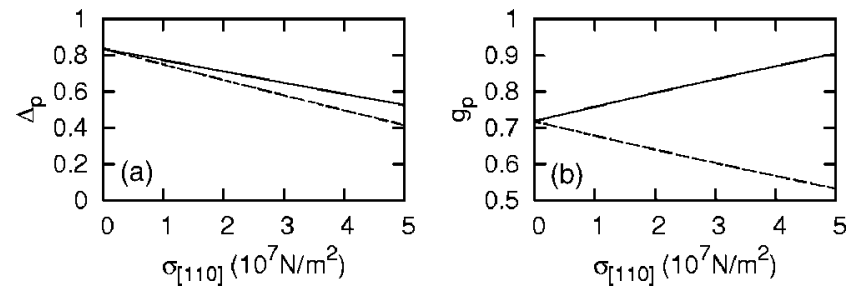

FIG. 2. (a) The gain peak position $\Delta_{p}$ as a function of applied stress $\sigma_{[110]} \cdot \Delta_{p,[1 \overline{10}]}$ is plotted as a solid line, while $\Delta_{p,[110]}$ is dashed. (b) The gain peak $g_{p}$ as a function of applied stress $\sigma_{[110]}$. $g_{p,[1 \overline{10}]}$ is plotted as a solid line, while $g_{p,[110]}$ is dashed. $D=1.5$ and all other parameters are as in Table III.

maximum becomes larger for $g_{[1 \overline{1} 0]}$, while the other goes down.

The differential gain at the gain peak can be found in Fig. 3. In Fig. 3(a), we see that the dependence on carrier density of the differential gain is typical. The transparency carrier density is lower for $g_{[110]}$ then for $g_{[110]}$ and the differential gain is also higher. In Fig. 3(b), we have plotted the differential gain as a function of applied stress. The differential gains split with the one related to [1 $\overline{1} 0]$ being the larger. Lastly, the transparency carrier density is plotted in Fig. 3(c). It is clear that the transparency carrier density for $g_{[1 \overline{1} 0]}$ becomes lower, while the other becomes larger. This is in correspondence with all prior results. Similarly, this theory also predicts a lower threshold current for the mode favored by stress. This effect is also observed experimentally [22].

\section{CARRIER-INDUCED DISPERSION AND LINEWIDTH ENHANCEMENT FACTOR}

Another important characteristic of semiconductor lasers is the strong dispersive effect accompanying material gain.
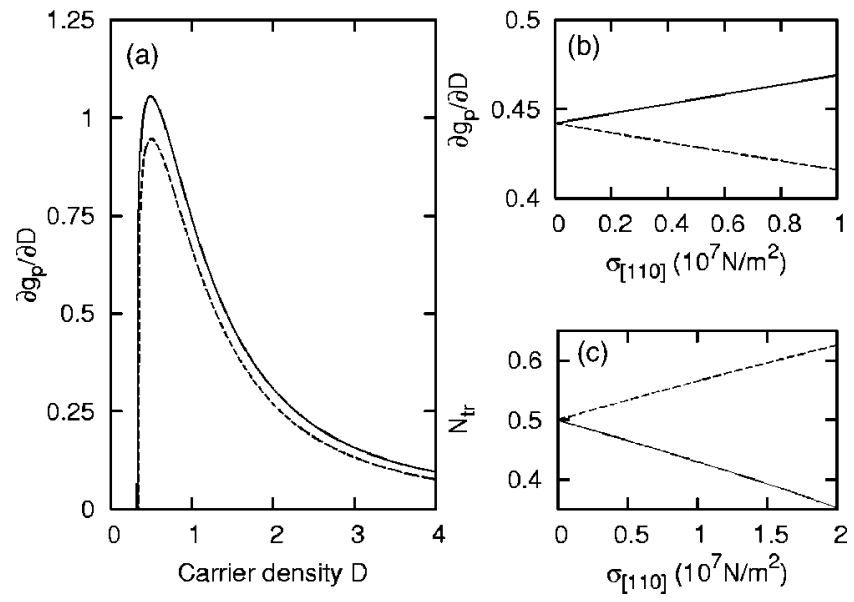

FIG. 3. (a) Differential gain at gain peak as a function of carrier density and for $\sigma_{[110]}=10^{7}$. (b) Differential gain at gain peak as a function of stress and $D=1.5$. The differential gain related to [1 $\overline{1} 0]$ is a solid line, while the one related to [110] is dashed. (c) The transparency carrier density as a function of stress. All other parameters are as in Table III.
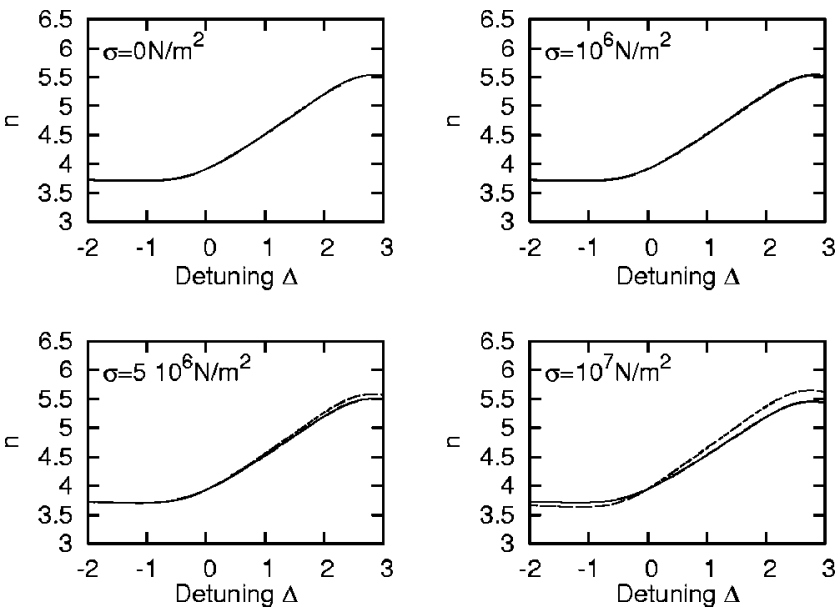

FIG. 4. Refractive index spectra for different values of $\sigma_{[110]}$. $n_{[110]}$ is plotted as a solid line, while $n_{[110]}$ is dashed. $D=1.5$ and all other parameters are as in Table III.

The refractive index change is associated with the real part of the susceptibility.

In Fig. 4, we have plotted the refractive index spectra. It can be seen that the refractive index spectra become polarization dependent. We can say that for the detuning region, where there is positive gain, the refractive index experienced by an electric field polarized along [1 $\overline{1} 0]$ will be smaller with respect to the orthogonal polarization. This large dispersive effect leads to a high degree of coupling between amplitude and frequency modulation. The latter effect is described by Henry's linewidth enhancement factor $\alpha$, which describes the changes in refractive index of the system with respect to the changes in the gain as the carrier density $D$ varies:

$$
\alpha(\Delta, D)=\frac{\operatorname{Re}(\partial \chi / \partial D)}{\operatorname{Im}(\partial \chi / \partial D)} .
$$

The linewidth enhancement factor will also split up for the two polarizations when stress is applied, with a higher $\alpha$ for the mode polarized along the direction of stress (see Fig. 5).

\section{SUMMARY}

We have studied the effect of uniaxially planar stress on the gain and refractive index spectra of a quantum well. To simplify the microscopic theory and to be able to obtain an analytical expression for the optical susceptibility, we have made the approximation that the band mixing contributions are small in second order. Assuming equal spin populations, our results not only showed that the eigenmodes associated with the quantum well become linearly polarized along the direction of stress, but we have found that the quantum well gain for the mode polarized perpendicular to the stress direction experiences higher gain that the one along the direction of stress. As a result, the transparency carrier density for the mode polarized perpendicular to the direction of stress is lower. In this way, it is possible to lower the threshold current by applying stress to the VCSEL package. Splitting of the gain curves leads to a splitting in the refractive index 

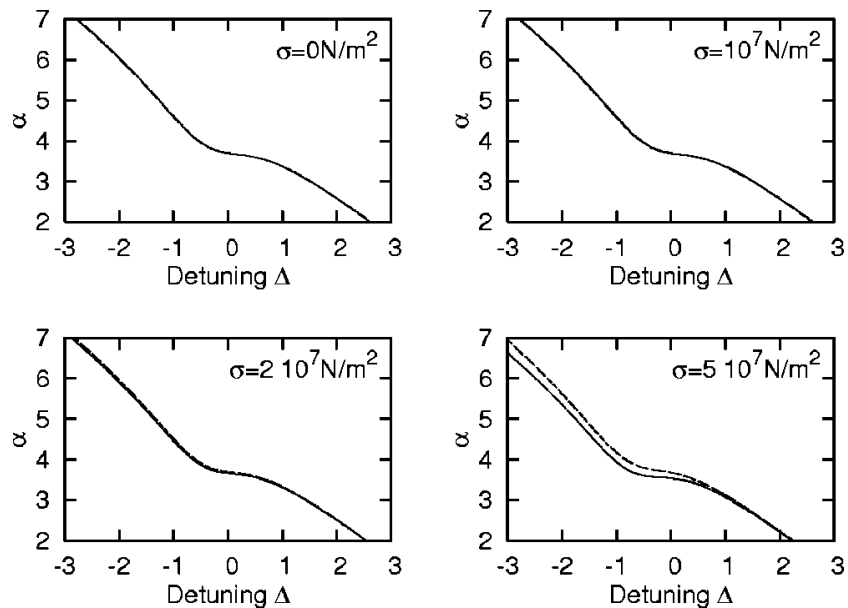

FIG. 5. The linewidth enhancement factor for different values of $\sigma_{[110]} \cdot \alpha_{[110]}$ is plotted as a solid line, while $\alpha_{[110]}$ is dashed. $D$ $=1.5$ and all other parameters are as in Table III.

spectra and linewidth enhancement factor. Generalization of this work to study dynamics of a diode laser system under stress is in progress.

\section{ACKNOWLEDGMENTS}

The authors acknowledge the European RTN network VISTA (Contract No. HPRN-CT-2000-00034) and the COST 288 action. J. D. and G. V. acknowledge the FWO (Fund for Scientific Research-Flanders) for their fellowships and for project support. On the Belgian side this work was supported by the Interuniversity Attraction Pole program (IAP V/18), the Concerted Research Action "Photonics in computing" and the Research Council of the Vrije Universiteit Brussel. S. B. acknowledges financial support from Ministerio de Ciencia y Tecnología (Spain) through project TIC2002-04255C04-03.

\section{APPENDIX A: DERIVATION OF THE HAMILTONIAN}

The Hamiltonian of the QW system, including the interaction with a semiclassical external optical field $\mathbf{E}$, reads [24]

$$
\begin{aligned}
\hat{H}= & H_{0}-\hat{\mathbf{P}} \cdot \mathbf{E} \\
= & \sum_{\mathbf{k}, s} E^{c}(k) a_{\mathbf{k}, s}^{\dagger} a_{\mathbf{k}, s} \\
& +\sum_{\mathbf{k}, j, j^{\prime}} T_{j j^{\prime}}(\mathbf{k}) b_{\mathbf{k}, j}^{\dagger} b_{\mathbf{k}, j^{\prime}} \\
& -\mathbf{E} \cdot \sum_{\mathbf{k}, s, j}\left(\mu_{s, j} a_{\mathbf{k}, s^{\dagger}} b_{\mathbf{k}, j}^{\dagger}+\text { h.a. }\right),
\end{aligned}
$$

where $a_{\mathbf{k}, s}\left(a_{\mathbf{k}, s}^{\dagger}\right)$ is the annihilation (creation) operator for electrons in the conduction band states $|\mathbf{k} s\rangle$ with in-plane wave vector $\mathbf{k}$ and spin $s= \pm 1 / 2$, which have energies $E^{c}(k)$. $T_{j j^{\prime}}(\mathbf{k})$ is the matrix element of the Hamiltonian between states in the valence band, $|\mathbf{k} j\rangle$, where $j= \pm 3 / 2, \pm 1 / 2$ denotes the $z$-component of the valence band angular momentum, and $b_{\mathbf{k}, j}\left(b_{\mathbf{k}, j}^{\dagger}\right)$ is the corresponding annihilation (creation) operator for holes in the valence band. The valence and conduction band states are coupled when an optical field is applied; assuming momentum conservation, the interaction among them is described by $\boldsymbol{\mu}_{s, j}$, the matrix element of the electric dipole moment between state $|\mathbf{k} s\rangle$ and $|\mathbf{k} j\rangle$, and h.a. denotes the hermitian adjoint. The selection rules impose that the only nonvanishing dipole moments are $\boldsymbol{\mu}_{1 / 2,3 / 2}, \boldsymbol{\mu}_{1 / 2,-1 / 2}$, $\boldsymbol{\mu}_{-1 / 2,1 / 2}$, and $\boldsymbol{\mu}_{-1 / 2,-3 / 2}[29]$ :

$$
\begin{gathered}
\boldsymbol{\mu}_{1 / 2,3 / 2}=\mu \mathbf{e}_{+}, \\
\boldsymbol{\mu}_{1 / 2,-1 / 2}=\frac{\mu}{\sqrt{3}} \mathbf{e}_{-}, \\
\boldsymbol{\mu}_{-1 / 2,1 / 2}=\frac{\mu}{\sqrt{3}} \mathbf{e}_{+}, \\
\boldsymbol{\mu}_{-1 / 2,-3 / 2}=\mu \mathbf{e}_{-} .
\end{gathered}
$$

If the in-plane component of the carriers' wave vector is small with respect to the orthogonal one (which is in the direction of the well), the band energies can be calculated using a perturbation analysis (k·p theory). In this approximation, the wave vector dependence of $\boldsymbol{\mu}_{s, j}$ can be neglected. In addition, the energy of the states in the conduction band is given by

$$
E^{c}(k)=E_{g}+E_{0 e}+\left(\hbar^{2} \gamma / 2 m_{0}\right) k^{2}+a_{e}\left(\varepsilon_{x x}+\varepsilon_{y y}+\varepsilon_{z z}\right),
$$

where $E_{g}$ is the gap energy, $E_{0 e}$ is the conduction band offset, and $m_{0} / \gamma$ is the effective mass in the conduction band states. The valence band energies can be calculated from the Luttinger Hamiltonian. In the basis of the electronic angular momentum eigenstates at band edge this Hamiltonian is block diagonal [24]

$$
T=\left(\begin{array}{cccc}
H^{h} & C & 0 & 0 \\
C^{*} & H^{l} & 0 & 0 \\
0 & 0 & H^{l} & C \\
0 & 0 & C^{*} & H^{h}
\end{array}\right),
$$

where, in the Pikus-Bir approximation [25], $H^{h}, H^{l}$, and $C$ are given by

$$
\begin{gathered}
H^{h}(k)=E_{0 h h}+\frac{\hbar^{2}}{2 m_{0}}\left(\gamma_{1}+\gamma_{2}\right) k^{2}+\varepsilon_{z z}\left(a_{1}-2 a_{2}\right)+\left(\varepsilon_{x x}+\varepsilon_{y y}\right) \\
\quad \times\left(a_{1}+a_{2}\right) \\
H^{l}(k)=E_{0 l h}+\frac{\hbar^{2}}{2 m_{0}}\left(\gamma_{1}-\gamma_{2}\right) k^{2}+\varepsilon_{z z}\left(a_{1}+2 a_{2}\right) \\
+\left(\varepsilon_{x x}+\varepsilon_{y y}\right)\left(a_{1}-a_{2}\right),
\end{gathered}
$$




$$
\begin{aligned}
C(k)= & -\frac{\sqrt{3} \hbar^{2}}{2 m_{0}}\left[\gamma_{2}\left(k_{x}^{2}-k_{y}^{2}\right)-2 i \gamma_{3} k_{x} k_{y}\right]-\sqrt{3} a_{2}\left(\varepsilon_{x x}-\varepsilon_{y y}\right) \\
& +2 \sqrt{3} i a_{3} \varepsilon_{x y} .
\end{aligned}
$$

In these equations, $a_{1}$ represents the hydrostatic deformation potential, $a_{2}$ and $a_{3}$ are the shear deformation potentials, and $\varepsilon_{i j}$ are elements of the strain tensor. $x, y$, and $z$ denote the main crystallographic axes whose orientations define the [100], [010], and [001] directions of the III-V semiconductor crystal, respectively.

In the experiments, tensile stress is applied to the VCSEL structure in the [110] and [1 $\overline{10}]$ directions and there is some residual stress present in the growth direction $([001])$. The stress tensor written in the base of the directions of applied stress reads

$$
\overline{\bar{\sigma}}=\left(\begin{array}{ccc}
\sigma_{[1 \overline{1} 0]} & 0 & 0 \\
0 & \sigma_{[110]} & 0 \\
0 & 0 & \sigma_{z}
\end{array}\right) .
$$

Hence, in the the crystallographic base $\{x, y, z\}$ it becomes

$$
\overline{\bar{\sigma}}_{C C S}=R \overline{\bar{\sigma}} R^{\dagger}=\left(\begin{array}{ccc}
\frac{\sigma_{[110]}+\sigma_{[1 \overline{1} 0]}}{2} & \frac{\sigma_{[110]}-\sigma_{[1 \overline{1} 0]}}{2} & 0 \\
\frac{\sigma_{[110]}-\sigma_{[1 \overline{1} 0]}}{2} & \frac{\sigma_{[110]}+\sigma_{[1 \overline{1} 0]}}{2} & 0 \\
0 & 0 & \sigma_{z}
\end{array}\right) .
$$

Using the compliance tensor $\left(S_{i j}\right)[30]$ it is possible to calculate the components of the strain tensor:

$$
\left(\begin{array}{c}
\varepsilon_{x x} \\
\varepsilon_{y y} \\
\varepsilon_{z z} \\
2 \varepsilon_{y z} \\
2 \varepsilon_{x z} \\
2 \varepsilon_{x y}
\end{array}\right)=\left(\begin{array}{c}
\frac{1}{2}\left(S_{11}+S_{12}\right)\left(\sigma_{[110]}+\sigma_{[1 \overline{1} 0]}\right)+S_{12} \sigma_{z} \\
\frac{1}{2}\left(S_{11}+S_{12}\right)\left(\sigma_{[110]}+\sigma_{[1 \overline{10}]}\right)+S_{12} \sigma_{z} \\
S_{12}\left(\sigma_{[110]}+\sigma_{[1 \overline{1} 0]}\right)+S_{11} \sigma_{z} \\
0 \\
0 \\
\frac{1}{2} S_{44}\left(\sigma_{[110]}-\sigma_{[1 \overline{1} 0]}\right)
\end{array}\right) .
$$

From this easy calculation, we can see that the change in symmetry of the unit cell when applying stress in the [110] and $[1 \overline{1} 0]$ is due to a shear strain. Defining the stress potentials

$$
\begin{gathered}
A_{e}=\left(S_{11}+2 S_{12}\right) a_{e}, \\
A_{h}=S_{12}\left(2 a_{1}-a_{2}\right)+S_{11}\left(a_{1}+a_{2}\right), \\
A_{l}=S_{12}\left(2 a_{1}+a_{2}\right)+S_{11}\left(a_{1}-a_{2}\right),
\end{gathered}
$$

$$
A_{c}=\frac{\sqrt{3} a_{3}}{2} S_{44}
$$

we can rewrite the expressions (A7) and (A9)-(A11) as

$$
\begin{aligned}
& E^{c}(k)=E_{g}+E_{0 e}^{\prime}+\left(\hbar^{2} \gamma / 2 m_{0}\right) k^{2}+A_{e}\left(\sigma_{[110]}+\sigma_{[1 \overline{1} 0]}\right), \\
& H^{h}(k)=E_{0 h h}^{\prime}+\frac{\hbar^{2}}{2 m_{0}}\left(\gamma_{1}+\gamma_{2}\right) k^{2}+A_{h}\left(\sigma_{[110]}+\sigma_{[1 \overline{1} 0]}\right), \\
& H^{l}(k)=E_{0 l h}^{\prime}+\frac{\hbar^{2}}{2 m_{0}}\left(\gamma_{1}-\gamma_{2}\right) k^{2}+A_{l}\left(\sigma_{[110]}+\sigma_{[1 \overline{1} 0]}\right),
\end{aligned}
$$

$$
C(k)=-\frac{\sqrt{3} \hbar^{2}}{2 m_{0}}\left[\gamma_{2}\left(k_{x}^{2}-k_{y}^{2}\right)-2 i \gamma_{3} k_{x} k_{y}\right]+i A_{c}\left(\sigma_{[110]}-\sigma_{[110]}\right) .
$$

The effect of $\sigma_{z}$ is included in $E_{0 e}^{\prime}, E_{0 h h}^{\prime}$, and $E_{0 l h}^{\prime}$, making clear that its role is simply to shift the edges of the bands, indeed:

$$
\begin{gathered}
E_{0 e}^{\prime}=E_{0 e}+\left(S_{11}+2 S_{12}\right) a_{e} \sigma_{z} \\
E_{0 h h}^{\prime}=E_{0 h h}+\left[S_{11}\left(a_{1}-2 a_{2}\right)+\left(S_{11}+S_{12}\right)\left(a_{1}+a_{2}\right)\right] \sigma_{z}
\end{gathered}
$$

$$
E_{0 l h}^{\prime}=E_{0 l h}+\left[S_{11}\left(a_{1}+2 a_{2}\right)+\left(S_{11}+S_{12}\right)\left(a_{1}-a_{2}\right)\right] \sigma_{z} .
$$

A complete diagonalization of the Hamiltonian $\hat{H}_{0}$ in Eq. (A1) can be accomplished by a suitable change of basis from the pure heavy-and light-hole states to mixed states $|\mathbf{k} h \pm\rangle$ and $|\mathbf{k} l \pm\rangle$, which are the eigenstates of the Luttinger hamiltonian associated with the degenerate eigenvalues

$$
\begin{aligned}
& E^{h}(\mathbf{k})=\frac{1}{2}\left[H^{h}+H^{l}-\sqrt{\left(H^{l}-H^{h}\right)^{2}+4|C|^{2}}\right] \\
& E^{l}(\mathbf{k})=\frac{1}{2}\left[H^{h}+H^{l}+\sqrt{\left(H^{l}-H^{h}\right)^{2}+4|C|^{2}}\right] .
\end{aligned}
$$

These mixed states are referred to as the mixed heavy-hole and mixed light-hole states and are found to be [24,31]:

$$
\begin{aligned}
& |\mathbf{k} h+\rangle=\gamma_{\mathbf{k}}\left(\left|\mathbf{k} \frac{3}{2}\right\rangle+\beta_{\mathbf{k}}\left|\mathbf{k}-\frac{1}{2}\right\rangle\right), \\
& |\mathbf{k} l-\rangle=\gamma_{\mathbf{k}}\left(-\beta_{\mathbf{k}}^{*}\left|\mathbf{k} \frac{3}{2}\right\rangle+\left|\mathbf{k}-\frac{1}{2}\right\rangle\right), \\
& |\mathbf{k} l+\rangle=\gamma_{\mathbf{k}}\left(\left|\mathbf{k} \frac{1}{2}\right\rangle-\beta_{\mathbf{k}}\left|\mathbf{k}-\frac{3}{2}\right\rangle\right),
\end{aligned}
$$




$$
|\mathbf{k} h-\rangle=\gamma_{\mathbf{k}}\left(\beta_{\mathbf{k}}^{*}\left|\mathbf{k} \frac{1}{2}\right\rangle+\left|\mathbf{k}-\frac{3}{2}\right\rangle\right),
$$

where

$$
\begin{gathered}
\beta_{\mathbf{k}}=\frac{C^{*}}{E^{h}-H^{l}}=\frac{C^{*}}{H^{h}-E^{l}}, \\
\gamma_{\mathbf{k}}=\frac{1}{\sqrt{1+\left|\beta_{\mathbf{k}}\right|^{2}}} .
\end{gathered}
$$

The annihilation operators of the mixed states $c_{\mathbf{k}, l \pm}$ and $c_{\mathbf{k}, l \pm}$ are

$$
\begin{aligned}
& c_{\mathbf{k}, h+}=\gamma_{\mathbf{k}}\left(b_{\mathbf{k}, 3 / 2}+\beta_{\mathbf{k}}^{*} b_{\mathbf{k},-1 / 2}\right), \\
& c_{\mathbf{k}, l-}=\gamma_{\mathbf{k}}\left(-\beta_{\mathbf{k}} b_{\mathbf{k}, 3 / 2}+b_{\mathbf{k},-1 / 2}\right), \\
& c_{\mathbf{k}, l+}=\gamma_{\mathbf{k}}\left(b_{\mathbf{k}, 1 / 2}-\beta_{\mathbf{k}}^{*} b_{\mathbf{k},-3 / 2}\right), \\
& c_{\mathbf{k}, h-}=\gamma_{\mathbf{k}}\left(\beta_{\mathbf{k}} b_{\mathbf{k}, 1 / 2}+b_{\mathbf{k},-3 / 2}\right) .
\end{aligned}
$$

while the mixed dipole moments become

$$
\begin{aligned}
& \mathbf{d}_{\mathbf{k}, h+}=\gamma_{\mathbf{k}} \mu\left(\mathbf{e}_{+}+\frac{\beta_{\mathbf{k}}^{*}}{\sqrt{3}} \mathbf{e}_{-}\right), \\
& \mathbf{d}_{\mathbf{k}, l-}=\gamma_{\mathbf{k}} \mu\left(-\beta_{\mathbf{k}} \mathbf{e}_{+}+\frac{\mathbf{e}_{-}}{\sqrt{3}}\right), \\
& \mathbf{d}_{\mathbf{k}, l+}=\gamma_{\mathbf{k}} \mu\left(\frac{\mathbf{e}_{+}}{\sqrt{3}}-\beta_{\mathbf{k}}^{*} \mathbf{e}_{-}\right) \\
& \mathbf{d}_{\mathbf{k}, h-}=\gamma_{\mathbf{k}} \mu\left(\frac{\beta_{\mathbf{k}}}{\sqrt{3}} \mathbf{e}_{+}+\mathbf{e}_{-}\right)
\end{aligned}
$$

Therefore, the total Hamiltonian can now be rewritten as

$$
\begin{aligned}
\hat{H}= & \sum_{\mathbf{k}, s} E^{c}(\mathbf{k}) a_{\mathbf{k}, s}^{\dagger} a_{\mathbf{k}, s}+\sum_{\mathbf{k}, \sigma} E^{\sigma}(\mathbf{k})\left(c_{\mathbf{k}, \sigma+}^{\dagger} c_{\mathbf{k}, \sigma+}+c_{\mathbf{k}, \sigma-}^{\dagger} c_{\mathbf{k}, \sigma-}\right) \\
& -\hat{\mathbf{P}} \cdot \mathbf{E},
\end{aligned}
$$

where $\sigma=\{h, l\}$ denotes the mixed heavy-hole and light-hole states. The interaction with the optical field is determined by the optical polarization of the QW medium,

$$
\begin{aligned}
\hat{\mathbf{P}}= & \sum_{\mathbf{k}}\left(\mathbf{d}_{\mathbf{k}, h+} a_{\mathbf{k}, 1 / 2}^{\dagger} c_{\mathbf{k}, h+}^{\dagger}+\mathbf{d}_{\mathbf{k}, l-l} a_{\mathbf{k}, 1 / 2}^{\dagger} c_{\mathbf{k}, l-}^{\dagger}+\mathbf{d}_{\mathbf{k}, l+} a_{\mathbf{k},-1 / 2}^{\dagger} c_{\mathbf{k}, l+}^{\dagger}\right. \\
& \left.+\mathbf{d}_{\mathbf{k}, h-} a_{\mathbf{k},-1 / 2}^{\dagger} c_{\mathbf{k}, h-}^{\dagger}\right)+ \text { h.a., }
\end{aligned}
$$

which describes the optical response of the system.

\section{APPENDIX B: DERIVATION OF THE OPTICAL SUSCEPTIBILITY}

We introduce the slowly varying envelopes of the electric field and of the microscopic polarizations, defined by

$$
\mathbf{E}=\widetilde{\mathbf{E}} e^{-i \omega t}+\text { c.c. },
$$

$$
\left\langle c_{\mathbf{k}, h \pm} a_{\mathbf{k}, \pm 1 / 2}\right\rangle=p_{\mathbf{k}, h \pm} e^{-i \omega t},
$$

and the carrier populations

$$
\begin{gathered}
n_{\mathbf{k}, \pm 1 / 2}^{e}=\left\langle a_{\mathbf{k}, \pm 1 / 2}^{\dagger} a_{\mathbf{k}, \pm 1 / 2}\right\rangle, \\
n_{\mathbf{k}, h \pm}=\left\langle c_{\mathbf{k}, h \pm}^{\dagger} c_{\mathbf{k}, h \pm}\right\rangle .
\end{gathered}
$$

In the slowly varying and rotating wave approximations, the Heisenberg equations for these quantities become [31]

$$
\begin{aligned}
\frac{d n_{\mathbf{k}, \pm 1 / 2}^{e}}{d t}= & \Lambda_{\mathbf{k}, \pm 1 / 2}-\frac{n_{\mathbf{k}, \pm 1 / 2}^{e}-f_{\mathbf{k}, \pm 1 / 2}^{e}}{\tau_{e}}-\frac{n_{\mathbf{k}, \pm 1 / 2}^{e}-n_{\mathbf{k}, \mp 1 / 2}^{e}}{\tau_{j e}} \\
& +i \Omega_{\mathbf{k}, h \pm} p_{k, h \pm}^{*}+\text { c.c. }, \\
\frac{d n_{\mathbf{k}, h \pm}}{d t}= & \Lambda_{\mathbf{k}, h \pm}-\frac{n_{\mathbf{k}, h \pm}-f_{\mathbf{k}, h \pm}}{\tau_{h}}-\frac{n_{\mathbf{k}, h \pm}-n_{\mathbf{k}, h \mp}}{\tau_{j h}}+i \Omega_{\mathbf{k}, h \pm} p_{k, h \pm}^{*} \\
& + \text { c.c. },
\end{aligned}
$$

$\frac{d p_{\mathbf{k}, h \pm}}{d t}=-\left[\gamma_{\perp}+i\left(\omega_{\mathbf{k}}^{h}-\omega\right)\right] p_{\mathbf{k}, h \pm}-i \Omega_{\mathbf{k}, h \pm}\left(n_{\mathbf{k}, h \pm}+n_{\mathbf{k}, \pm 1 / 2}^{e}-1\right)$.

Here, we have phenomenologically included the pump rates of each state $(\Lambda)$, the relaxation terms describing the evolution of the carrier distribution to their quasi-equilibrium value (the Fermi distributions for electrons $f_{\mathbf{k}, \pm 1 / 2}^{e}$ and holes $f_{\mathbf{k}, h \pm}$ ) with time constants $\tau_{e}$ and $\tau_{h}$, respectively, the spin-flip processes that mix the states within the valence and conduction bands with time scales $\tau_{j}$, and the dephasing of the dipoles due to intraband carrier-carrier scattering with characteristic rate $\gamma_{\perp}$. We have also defined the transition frequencies $\hbar \omega_{\mathbf{k}}^{h}=E^{c}(k)+E^{h}(\mathbf{k})$ and the Rabi frequencies $\hbar \Omega_{\mathbf{k}, h \pm}=\mathbf{d}_{\mathbf{k}, h \pm} \cdot \widetilde{\mathbf{E}}$. By using the basis of circularly polarized light states, $\widetilde{\mathbf{E}}=E_{+} \mathbf{e}_{+}+E_{-} \mathbf{e}_{-}$and noting that $\mathbf{e}_{ \pm} \cdot \mathbf{e}_{ \pm}=0, \mathbf{e}_{ \pm} \cdot \mathbf{e}_{\overline{+}}$ $=-1$, and $\mathbf{e}_{ \pm}=-\mathbf{e}_{\mp}^{*+1}$, the Rabi frequencies read

$$
\begin{aligned}
& \hbar \Omega_{\mathbf{k}, h+}=-\gamma_{\mathbf{k}} \mu\left(E_{-}+\frac{\beta_{\mathbf{k}}^{*}}{\sqrt{3}} E_{+}\right), \\
& \hbar \Omega_{\mathbf{k}, h-}=-\gamma_{\mathbf{k}} \mu\left(E_{+}+\frac{\beta_{\mathbf{k}}}{\sqrt{3}} E_{-}\right) .
\end{aligned}
$$

Due to the large intraband carrier-carrier scattering rates, the carrier populations are driven into the quasi-equilibrium Fermi distributions within very short time scales $(\sim 0.1 \mathrm{ps})$. the same is true for the microscopic polarizations. Hence, we have that the microscopic polarizations are stationary and that (B7) becomes

\footnotetext{
${ }^{\mathrm{l}}$ These expressions might seem contradictory to the definition of the scalar product in a two-dimensional Hilbert space. However, this scalar product has to be interpreted within the domain of normal Euclidian space.
} 


$$
p_{\mathbf{k}, h \pm}=-i \Omega_{\mathbf{k}, h \pm} \frac{f_{\mathbf{k}, h \pm}+f_{\mathbf{k}, \pm 1 / 2}^{e}-1}{\gamma_{\perp}+i\left(\omega_{\mathbf{k}}^{h}-\omega\right)} .
$$

The macroscopic polarization $\mathbf{P}$ can be calculated using

$$
\mathbf{P}=\frac{1}{V} \sum_{\mathbf{k}}\left(\mathbf{d}_{\mathbf{k}, h+}^{*} p_{\mathbf{k}, h+}+\mathbf{d}_{\mathbf{k}, h-}^{*} p_{\mathbf{k}, h-}\right) .
$$

There are four different contributions:

$$
\begin{gathered}
\mathbf{P}_{h+,+}=-\frac{i \mu^{2}}{\hbar V} \sum_{\mathbf{k}}\left|\gamma_{\mathbf{k}}\right|^{2} \frac{f_{\mathbf{k}, h+}+f_{\mathbf{k},+1 / 2}^{e}-1}{\gamma_{\perp}+i\left(\omega_{\mathbf{k}}^{h}-\omega\right)}\left(\frac{\left|\beta_{\mathbf{k}}\right|^{2}}{3} E_{+}+\frac{\beta_{\mathbf{k}}^{*}}{\sqrt{3}} E_{-}\right) \mathbf{e}_{+}, \\
\mathbf{P}_{h+,-}=-\frac{i \mu^{2}}{\hbar V} \sum_{\mathbf{k}}\left|\gamma_{\mathbf{k}}\right|^{2} \frac{f_{\mathbf{k}, h+}+f_{\mathbf{k},+1 / 2}^{e}-1}{\gamma_{\perp}+i\left(\omega_{\mathbf{k}}^{h}-\omega\right)}\left(\frac{\beta_{\mathbf{k}}}{\sqrt{3}} E_{+}+E_{-}\right) \mathbf{e}_{-}, \\
\mathbf{P}_{h-,+}=-\frac{i \mu^{2}}{\hbar V} \sum_{\mathbf{k}}\left|\gamma_{\mathbf{k}}\right|^{2} \frac{f_{\mathbf{k}, h-}+f_{\mathbf{k},-1 / 2}^{e}-1}{\gamma_{\perp}+i\left(\omega_{\mathbf{k}}^{h}-\omega\right)}\left(E_{+}+\frac{\beta_{\mathbf{k}}^{*}}{\sqrt{3}} E_{-}\right) \mathbf{e}_{+}, \\
\mathbf{P}_{h-,-}=-\frac{i \mu^{2}}{\hbar V} \sum_{\mathbf{k}}\left|\gamma_{\mathbf{k}}\right|^{2} \frac{f_{\mathbf{k}, h-}+f_{\mathbf{k},-1 / 2}^{e}-1}{\gamma_{\perp}+i\left(\omega_{\mathbf{k}}^{h}-\omega\right)}\left(\frac{\beta_{\mathbf{k}}}{\sqrt{3}} E_{+}+\frac{\left|\beta_{\mathbf{k}}\right|^{2}}{3} E_{-}\right) \mathbf{e}_{-},
\end{gathered}
$$

where $\mathbf{P}_{b, p}$ indicates a contribution to the left $(p=-)$ or right $(p=+)$ polarized polarization field induced by the transition between conduction band and mixed heavy-hole band with either positive $(b=h+)$ or negative spin $(b=h-)$. By using the standard definition of the optical susceptibility $\mathbf{P}=\varepsilon_{0} \overline{\bar{\chi}} \mathbf{E}$, it can be identified from Eqs. (B12)-(B15).

\section{APPENDIX C: DERIVATION OF THE APPROXIMATED SUSCEPTIBILITY}

The unmixed susceptibility $G_{ \pm}$given in Eq. (15) can be split into three different contributions, $G=G_{e}+G_{h}-G_{b}$, i.e.,

$$
\begin{gathered}
G_{e \pm}=\frac{\mu^{2}}{\varepsilon_{0} V \hbar} \sum_{k} \frac{f_{k, e \pm}}{\omega-\omega_{k}^{h}+i \gamma_{\perp}}, \\
G_{h \pm}=\frac{\mu^{2}}{\varepsilon_{0} V \hbar} \sum_{k} \frac{f_{k, h \pm}}{\omega-\omega_{k}^{h}+i \gamma_{\perp}}, \\
G_{b}=\frac{\mu^{2}}{\varepsilon_{0} V \hbar} \sum_{k} \frac{1}{\omega-\omega_{k}^{h}+i \gamma_{\perp}} .
\end{gathered}
$$

Proceeding as in [16], we can perform the summations over the in-plane wave vector, leading to

$$
\begin{aligned}
& G_{e \pm}(\omega)=-\frac{\chi_{0}}{2} \ln \left(1-\frac{2 D_{e \pm}}{u+i}\right), \\
& G_{h \pm}(\omega)=-\frac{\chi_{0}}{2} \ln \left(1-\frac{2 D_{h \pm}}{u+i}\right),
\end{aligned}
$$

$$
G_{b}(\omega)=-\frac{\chi_{0}}{2} \ln \left(1-\frac{b}{u+i}\right)
$$

where

$$
\chi_{0}=\frac{m \mu^{2}}{\varepsilon_{0} W \pi \hbar^{2}}
$$

$$
\begin{array}{r}
b=\frac{\hbar}{2 m \gamma_{\perp}} k_{b}^{2}, \\
u=\frac{\omega-\frac{E_{t}}{\hbar}}{\gamma_{\perp}} .
\end{array}
$$

In the above equations, $W$ is the thickness of the $\mathrm{QW}$. The reduced mass is $m=m_{0} /\left(\gamma+\gamma_{1}+\gamma_{2}\right) . b$ measures, in units of $\gamma_{\perp}$, the total energy spread where optical transitions from the the heavy-hole and to the conduction band may occur; thus $G_{b}(\omega)$ describes the optical response of the system when there are no carriers in the bands. Finally, $u$ measures the detuning of the optical field with the minimum transition in units of $\gamma_{\perp}$, and $D_{e(h) \pm}$ stand for the densities of electrons (holes) in each spin orientation normalized to the transparency carrier density,

$$
\begin{gathered}
D_{e(h) \pm}=\frac{N_{e(h) \pm}}{N_{t}}, \\
N_{t}=\frac{m \gamma_{\perp}}{\pi \hbar W} .
\end{gathered}
$$

Therefore, the unmixed susceptibility is finally given by

$$
G_{ \pm}(u)=-\frac{\chi_{0}}{2} \ln \left(\frac{\left(u+i-2 D_{e \pm}\right)\left(u+i-2 D_{h \pm}\right)}{(u+i)(u+i-b)}\right) .
$$

The nondiagonal part of the susceptibility, $S_{ \pm}$is given by Eq. (22). Again, it can be split into three different contributions, $S=S_{e}+S_{h}-S_{b}$, i.e.,

$$
\begin{aligned}
& S_{e \pm}=i \frac{\mu^{2}}{\sqrt{3} V \hbar \varepsilon_{0}} \sum_{k} \frac{f_{k, e \pm}}{\omega-\omega_{k}^{h}+i \gamma_{\perp}} \frac{A_{c}\left(\sigma_{[110]}-\sigma_{[1 \overline{10}]}\right)}{E^{h}-H^{l}}, \\
& S_{h \pm}=i \frac{\mu^{2}}{\sqrt{3} V \hbar \varepsilon_{0}} \sum_{k} \frac{f_{k, h \pm}}{\omega-\omega_{k}^{h}+i \gamma_{\perp}} \frac{A_{c}\left(\sigma_{[110]}-\sigma_{[1 \overline{10}]}\right)}{E^{h}-H^{l}},
\end{aligned}
$$

$$
S_{b}=i \frac{\mu^{2}}{\sqrt{3} V \hbar \varepsilon_{0}} \sum_{k} \frac{1}{\omega-\omega_{k}^{h}+i \gamma_{\perp}} \frac{A_{c}\left(\sigma_{[110]}-\sigma_{[110]}\right)}{E^{h}-H^{l}} .
$$

To first order in band mixing, we have that 


$$
\begin{aligned}
E^{h}-H^{l} \approx & H^{h}-H^{l}=E_{0 h h}^{\prime}-E_{0 l h}^{\prime}+\left(A_{h}-A_{l}\right)\left(\sigma_{[110]}+\sigma_{[110]}\right) \\
& +\frac{\hbar}{m_{0}} \gamma_{2} k^{2} .
\end{aligned}
$$

Hence, the nondiagonal part of the susceptibility can finally be computed, the result being

$$
S_{ \pm}(u)=i S_{0} \frac{\sigma_{[110]}-\sigma_{[110]} \bar{n}}{u-u_{\sigma}}\left[G_{ \pm}(u)-G_{ \pm}\left(u_{\sigma}\right)\right]
$$

where we have defined

$$
\begin{gathered}
S_{0}=\frac{A_{c}}{\sqrt{3} \hbar \gamma_{\perp}} \frac{\gamma+\gamma_{1}+\gamma_{2}}{2 \gamma_{2}}, \\
u_{\sigma}=-i-\frac{\gamma+\gamma_{1}+\gamma_{2}}{2 \gamma_{2}} \frac{E_{0 h h}^{\prime}-E_{0 l h}^{\prime}+\left(A_{h}-A_{l}\right)\left(\sigma_{[110]}+\sigma_{[1 \overline{10}]}\right)}{\hbar \gamma_{\perp}} \\
=-i-u_{\sigma 0}-u_{\sigma 1}\left(\sigma_{[110]}+\sigma_{[1 \overline{1} 0]}\right) .
\end{gathered}
$$

As a final step, we note that the spin relaxation rate for the

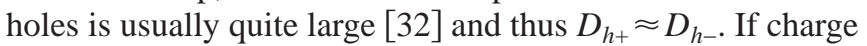
neutrality is assumed, $D_{e^{+}}+D_{e_{-}}=D_{h_{+}}+D_{h_{-}}$, we can replace the hole densities by

$$
D_{h \pm} \approx \frac{D_{e+}+D_{e-}}{2} .
$$

This approximation allows us to describe the optical response of the system, dependent on the densities of electrons in each spin orientation, hence, in the following we will drop the subscript $e$ in the electron populations $\left(D_{ \pm}=D_{e \pm}\right)$. We can now rewrite the susceptibility tensor as follows:

$$
\overline{\bar{\chi}}\left(D_{+}, D_{-}, u\right)=\left(\begin{array}{cc}
G_{-}(u) & S_{+}(u)+S_{-}(u) \\
-S_{+}(u)-S_{-}(u) & G_{+}(u)
\end{array}\right),
$$

with

$$
\begin{gathered}
G_{ \pm}(u)=-\frac{\chi_{0}}{2} \ln \left(\frac{\left(u+i-2 D_{ \pm}\right)\left(u+i-D_{+}-D_{-}\right)}{(u+i)(u+i-b)}\right), \\
S_{ \pm}(u)=i S_{0} \frac{\sigma_{[110]}-\sigma_{[110]}}{u-u_{\sigma}}\left[G_{ \pm}(u)-G_{ \pm}\left(u_{\sigma}\right)\right] .
\end{gathered}
$$

Last, we phenomenologically include the band-gap shrinkage due to many-body effects (the so-called band-gap renormalization), which leads to

$$
u=\Delta+\rho\left(D_{+}+D_{-}\right)^{1 / 3},
$$

where $\Delta=\left(\omega-\omega_{t}\right) / \gamma_{\perp}$ is the detuning between the optical frequency $\omega$ and the nominal transition frequency $\omega_{t}$ normalized to the linewidth, and $\rho$ describes the effective band-gap shrinkage with the total carrier density.
[1] K. D. Choquette, D. A. Richie, and R. E. Leibenguth, Appl. Phys. Lett. 64, 2062 (1994).

[2] B. Ryvkin, K. Panajotov, A. Georgievski, J. Danckaert, M. Peeters, G. Verschaffelt, H. Thienpont, and I. Veretennicoff, J. Opt. Soc. Am. B 16, 2106 (1999).

[3] K. Panajotov, B. Ryvkin, J. Danckaert, M. Peeters, H. Thienpont, and I. Veretennicoff, IEEE Photonics Technol. Lett. 10, 6 (1998).

[4] A. Valle, K. A. Shore, and L. Pesquera, J. Lightwave Technol. 14, 2062 (1996).

[5] Y. Takahashi and H. Kawaguchi, IEEE J. Quantum Electron. 36, 864 (2000).

[6] J. Albert, G. Van der Sande, B. Nagler, K. Panajotov, I. Veretennicoff, J. Danckaert, and T. Erneux, Opt. Commun. 248, 527 (2005).

[7] A. Valle, J. Sarma, and K. A. Shore, IEEE J. Quantum Electron. 31, 1423 (1995).

[8] B. M. Yu and J. M. Liu, J. Appl. Phys. 69, 7444 (1991).

[9] F. Prati, P. Caccia, and F. Castelli, Phys. Rev. A 66, 063811 (2002).

[10] S. Balle, Opt. Lett. 27, 1923 (2002).

[11] B. S. Ryvkin, E. A. Avrutin, and M. Pessa, J. Appl. Phys. 93, 2353 (2003).
[12] B. S. Ryvkin and A. M. Georgievskii, Semiconductors 33, 813 (1999).

[13] M. Grupen and K. Hess, Appl. Phys. Lett. 70, 808 (1997).

[14] M. San Miguel, Q. Feng, and J. V. Moloney, Phys. Rev. A 52, 1728 (1995).

[15] J. Martin-Regalado, F. Prati, M. San Miguel, and N. B. Abraham, IEEE J. Quantum Electron. 33, 765 (1997).

[16] S. Balle, Phys. Rev. A 57, 1304 (1998).

[17] S. Balle, E. Tolkachova, M. San Miguel, J. R. Tredicce, J. Martin-Regalado, and A. Gahl, Opt. Lett. 24, 1121 (1999).

[18] M. Sondermann, M. Weinkath, and T. Ackemann, IEEE J. Quantum Electron. 40, 97 (2004).

[19] M. Sondermann, M. Weinkath, T. Ackemann, J. Mulet, and S. Balle, Phys. Rev. A 68, 033822 (2003).

[20] M. Sondermann, T. Ackemann, S. Balle, J. Mulet, and K. Panajotov, Opt. Commun. 235, 421 (2004).

[21] M. P. van Exter, A. K. J. van Doorn, and J. P. Woerdman, Phys. Rev. A 56, 845 (1997).

[22] K. Panajotov, B. Nagler, G. Verschaffelt, A. Georgievski, H. Thienpont, J. Danckaert, and I. Veretennicoff, Appl. Phys. Lett. 77, 1590 (2000).

[23] K. Panajotov, B. Nagler, G. Verschaffelt, J. Albert, J. Danckaert, I. Veretennicoff, H. Thienpont, J. Yong, and J. Rorison, 
Proc. SPIE 4286, 55 (2001).

[24] W. W. Chow, S. W. Koch, and M. Sargent III, Semiconductor Laser Physics (Springer, Heidelberg, 1994).

[25] G. L. Bir and G. E. Pikus, Symmetry and Strain-Induced Effects in Semiconductors (Wiley and Sons, New York, 1974).

[26] D. Burak, J. V. Moloney, and R. Binder, Phys. Rev. A 61, 053809 (pages 21) (2000).

[27] D. Burak, J. V. Moloney, and R. Binder, IEEE J. Quantum Electron. 36, 956 (2000).
[28] I. Vurgaftman, J. R. Meyer, and L. R. Ram-Mohan, J. Appl. Phys. 89, 5815 (2001).

[29] L. A. Coldren and S. W. Corzine, Diode Lasers and Photonic Integrated Circuits (Wiley and Sons, New York, 1995).

[30] D. R. Lovett, Tensor Properties of Crystals (IOP, Bristol, 1989).

[31] F. Prati, L. Fratta, and M. Travagnin, Phys. Rev. A 62, 033819 (2000).

[32] L. Vina, J. Phys.: Condens. Matter 11, 5929 (1999). 